\title{
Collection Le Point sur... Réanimation pédiatrique
}

\author{
Olivier Paut, Gilles Orliaguet. Collection dirigée par J.L. Vincent et C. Martin. Springer; \\ 2013. ISBN 978-2-8178-0406-4
}

\author{
Marie-Andrée Girard, MD
}

Received: 27 April 2013/Accepted: 25 June 2013/Published online: 3 July 2013

(C) Canadian Anesthesiologists' Society 2013

La réalité des soins intensifs pédiatriques est loin de celle des soins intensifs adultes. Les pathologies, la réponse physiologique et l'environnement psychosocial nécessitent une approche particulière de l'équipe de soins. Dans les dernières années, de nombreux débats ont animé la scène dans le domaine des soins intensifs pédiatriques et on est toujours à la recherche d'une conclusion. Deux experts français en soins intensifs pédiatriques (appelé dans l'Hexagone «Réanimation Pédiatrique ») ont décidé récemment de rejoindre le collectif Le Point sur... et publient pour la première fois cette année les fruits d'un effort collectif impliquant de nombreux collaborateurs. Ce champ de connaissance, bien qu'il soit étroit, a encore beaucoup de potentiel de développement et ce travail français est accueilli avec un grand enthousiasme.

Dirigés par le Pr Gilles Orliaguet, de Paris, et le Pr Olivier Paut, de Marseille, de nombreux experts (plus de 40 auteurs ont participé à ce travail) proposent au lecteur un livre bien écrit. La structure de l'ouvrage est basée sur les problématiques cliniques et permet au lecteur de mettre pleinement ses connaissances à jour, tout en respectant une certaine unité. En fait, le lecteur ne découvrira pas dans ces pages un lourd livre de référence, mais plutôt un outil convivial d'actualisation de ses connaissances, ce qui cadre très bien avec la philosophie de la collection Le Point sur...

Nonobstant cette impression, ce livre n'est pas une suite de résumés de problématiques cliniques. Débutant par de

M.-A. Girard, MD (ه)

Département d'anesthésiologie, Hôpital Sainte-Justine,

Université de Montréal, Montréal, QC, Canada

e-mail: marie-andree.girard.1@umontreal.ca l'épidémiologie et de l'économie et se terminant avec l'éthique en fin de vie, les auteurs couvrent tous les fondements de soins intensifs en milieu pédiatrique: choc, arrêt cardiorespiratoire, syndrome de détresse respiratoire, ventilation, surveillance hémodynamique, insuffisance rénale, traumatismes, syndrome du compartiment abdominal... Chaque chapitre commence par une brève introduction suivie d'un texte dont la disposition est conçue en fonction du sujet. Même si les chapitres sont concis, les auteurs parviennent à présenter clairement la réalité globale de chaque sujet plutôt que de se limiter à la situation et aux prises en charge européennes. Chaque sujet est d'ailleurs présenté avec une grande quantité de détails et de références. En particulier dans les chapitres sur la surveillance hémodynamique et la sédation en réanimation, la présentation approfondie permet de bien comprendre la portée du sujet.

Le style choisi par les auteurs a toutefois un grand défaut: les illustrations, et en particulier les illustrations en couleur, sont sous-utilisées. Leur présence aurait souligné certains aspects du contenu et ainsi facilité la compréhension de certains concepts. Aussi, en cette ère des technologies, l'addition de matériel audiovisuel supplémentaire aurait satisfait le lecteur avide et permis de maximiser l'information donnée par les auteurs. Enfin, si vous désirez chercher un concept bien précis dans ce livre, préparez-vous à une tâche difficile. L'absence d'index, qu'il soit thématique ou sémantique, rend la recherche d'un sujet spécifique compliquée, particulièrement si vous ne savez pas dans quel chapitre il devrait se trouver.

Alors, à qui s'adresse ce livre? Le public visé inclut les spécialistes dans le domaine des soins intensifs pédiatriques ayant besoin d'une bonne synthèse sur des sujets précis, mais le livre pourrait être utile au résident ou 
à l'étudiant à la recherche d'un ouvrage facile à lire en français.

« Le Point sur... Réanimation Pédiatrique » est donc un bon ajout à votre bibliothèque scientifique. Sa portée est étroite mais chaque livre dans ce domaine a sa valeur intrinsèque et doit être considéré comme tel.

Conflit d'intérêt Aucun. 